\title{
Laparoscopic Repair of Perforated Duodenal Ulcer with Omental Patch
}

\author{
Mohamed Ibrahim Hassan, MD; Mohamed Shaaban Khalifa, MD.
}

\author{
Department of General Surgery, Ain Shams University, Cairo, Egypt.
}

Background: Duodenal perforation is a common complication of duodenal ulcer. Treatment for perforated ulcer range from conservative treatment approach to radical surgery. Laparscopic management could be done, making it possible to avoid a median laparotomy which can lead to wound infection and late incisional hernia. We present our experience with the laparoscopic management of the perforated duodenal ulcer.

Patients and methods: This study included 20 patients who underwent laparoscopic repair of perforated duodenal ulcer at our department. The patients were admitted in urgent setting. A detailed history was taken. The patients were well examined, and the time from the onset of symptoms to the beginning of the operation was recorded. The main diagnostic procedure performed was abdominal X-ray in erect position; in addition to abdominal ultrasound examination. Resuscitation was done preoperatively with I.V fluids and antibiotic.

Results: The average operative time was 50 min $( \pm 15)$. 18 patients were operated upon laparoscopically, however 2 patients were converted to open procedure. Only 2 morbidities occurred, 1 patient developed leakage postoperatively which healed on conservative management and the other had sever post operative pneumonia. The average hospital stay was 5 days with no mortalities.

Conclusion: Laparoscopic primary repair with omental patch is a good method for the surgical treatment of duodenal ulcer perforation, reducing hospital stay, complications and return to normal activity if carried on in a proper manner.

Key words: Perforated, peptic ulcer, laparoscopic repair, omental patch.

\section{Introduction:}

Peptic ulcer disease is still the major cause of gastrointestinal perforation, despite major improvements in both diagnostic and therapeutic strategies. ${ }^{1}$

The diagnosis of a perforated ulcer is straight forward when an acute onset of epigastric pain is observed in a patient with a known history of peptic ulcer disease. In such instances, radiological investigation is usually limited to plain abdominal X-ray films to document the associated pneumoperitoneum. ${ }^{2}$

Less commonly, clinical onset of a perforated gastric or duodenal ulcer may be atypical or subtle because of co-morbidities and/or concurrent therapies. ${ }^{3}$

Treatment for perforated ulcer ranges from conservative treatment (Taylor's approach) to radical surgery (vagotomy, gastrectomy). However, with the use of powerful acid suppressing medication and the eradication of Helicobacter pylori, the need for radical surgery in emergencies has sharply declined. The surgical technique most often used is the closure of the perforation combined with extensive peritoneal lavage. Repair of duodenal perforation by Graham patch plication (as was described in 1937) represents an excellent alternative approach. ${ }^{4}$

Treatment for perforated ulcer can be performed laparoscopically in $85 \%$ of cases, making it possible to avoid a median laparotomy which can lead to wound infection and late incisional hernia. 5

Recent papers suggest that laparoscopic 
repair is a safe and effective procedure.

In selected patients, offering shorter operating time, less postoperative pain and shorter postoperative hospital stay. ${ }^{6}$

\section{Study design:}

The aim of the current study is to present our experience with the laparoscopic repair of perforated duodenal ulcer.

\section{Patient and methods:}

The operation started with the insertion of a $10 \mathrm{~mm}$ umblical port (port 1) through the open technique to avoid any injury through the blind insertion of the Veress needle, that port was used for laparoscope. Pneumoperitoneum of $13 \mathrm{~mm} \mathrm{Hg}$ was achieved.

Under visual control two additional ports were placed, a $10 \mathrm{~mm}$ port approximately in the middle between the xiphoid process and umbilicus (port 2), slightly paramedian to the left and a $5 \mathrm{~mm}$ port in the right midclavicular line below the costal margin (port 3 ) and the port for liver retraction just below the xiphoid process slightly to the right (port 4).

Full exploration of the abdominal cavity was performed. Fine manipulation of the edematous bowel was respected and the site of the duodenal perforation was detected Figure (2). Sample of the peritoneal fluid was taken for culture and sensitivity. Toilet of the abdominal cavity was done by repeated irrigation and suction by warm normal saline (average 2 liters).

Perforation margins were identified, Intracorporeal suturing was done with $2 / 0$ Vicryl absorbable suture. We avoided the extracorporeal stitches as the edges of the perforation were friable and cannot withstand any traction.

Interrupted sutures were used and usually two or three stitches were placed in a transversal manner over the perforation focused on the pyloroduodenal axis.

The threads were cut long .Once the perforation was sealed, a small fragment of the greater omentum was fixed over the suture line using the upper thread.

18 F abdominal drain was positioned, through the right sub costal port to the right paracolic gutter.

Postoperative treatment protocol was carried out. The patients had received a proton pump inhibitor pantoprazol $40 \mathrm{mg} / 12 \mathrm{~h}$ intravenously in the hospital and orally after discharge for 4 weeks. Intravenous antibiotic therapy was third generation cephalosporins $1 \mathrm{gm}$ every 12 hours and metronidazole $500 \mathrm{mg}$ every 8 hours for the anaerobes, was maintained depending on the severity of the peritonitis. The antibiotic therapy was adjusted according to the culture and sensitivity results.

The nasogastric tube was removed once the peristalsis resumed and the intestinal sounds were audible. Water-soluble gastro esophageal contrast (GastrografinTM) examination was then performed on the $3 \mathrm{rd}$ day to check the integrity of the closure, to detect any sign of leakage and ensure the absence of pyloro-duodenal stenosis.

Food intake was restored on the third day post operative and the drain was removed once the effluent is less than $100 \mathrm{ml}$ per day and serous in nature.

Thromboprophylaxis with enoxaparin was administered mainly to the obese patients BMI more than 40 during the entire postoperative hospital stay.

Upper GIT endoscopy was performed usually 4 to 6 weeks after the operation for detection of any inflammation, erosion or gastric and duodenal ulcer and monitor the efficacy of the medical treatment.

\section{Results:}

This study included 20 patients (16 males and 4 females) between June 2012 and June 2014 who underwent laparoscopic repair of perforated duodenal ulcer.The median age was 37 years (19 55 years). Median BMI at the time of operation was $29.5(17 \sim 42)$ Table (1).

The average operative time was $50 \mathrm{~min}$ $( \pm 15) .20$ patients were operated upon laparoscopically. However 2 patients were converted to open procedure. One of them because the perforation was larger than 2 $\mathrm{cm}$ with marked peritonitis with multiple 


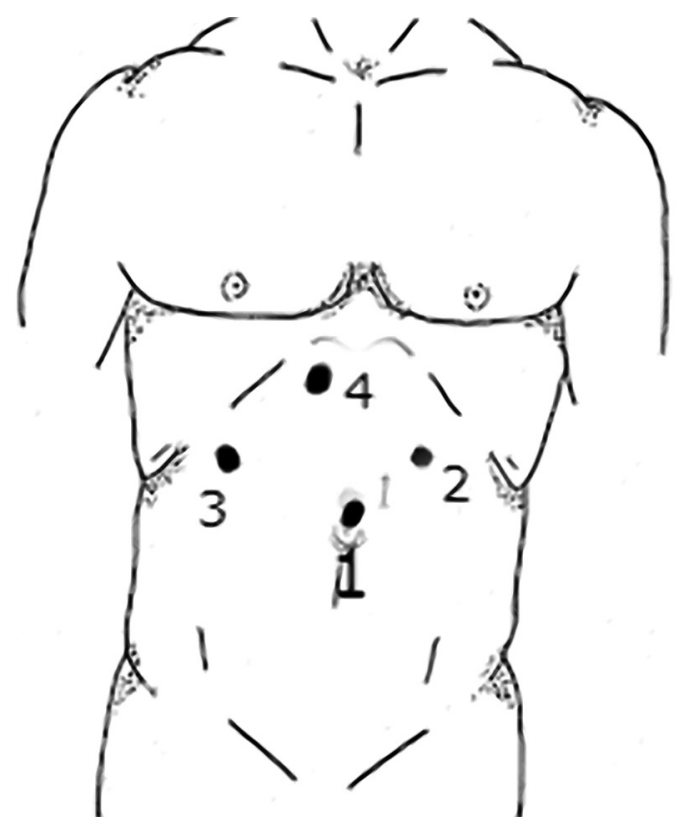

Figure (1): Port sites.

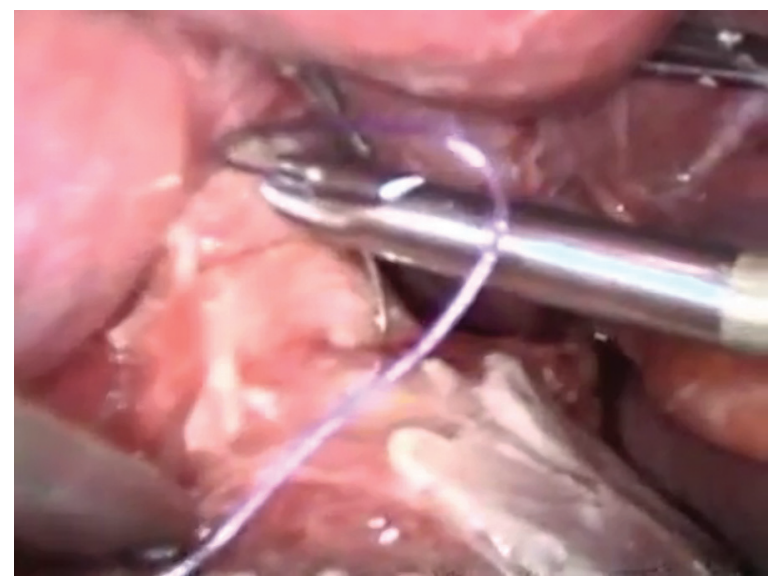

Figure (3): Intracorporeal stitching of the perforation.

abdominal and pelvic abscesses and the patient had previous abdominal surgery and marked adhesions. The other case was converted as recommended by the anesthesiologists stuff because of marked intraoperative respiratory distress Table (2).

The post-operative period passed smoothly for 18 patients with removal of the nasogastric tube once the intestinal sounds were audible. Oral feeding was started on the 3rd day post operative, after which the gastrograffin study was done, the hospital stay was $5( \pm 2)$.

We had experienced complications in 2 patients. The first was 35 year male patient, on the 3rd day gastrograffin study was done, showed leak that was noticed also at the color of the drains. Conservative management was

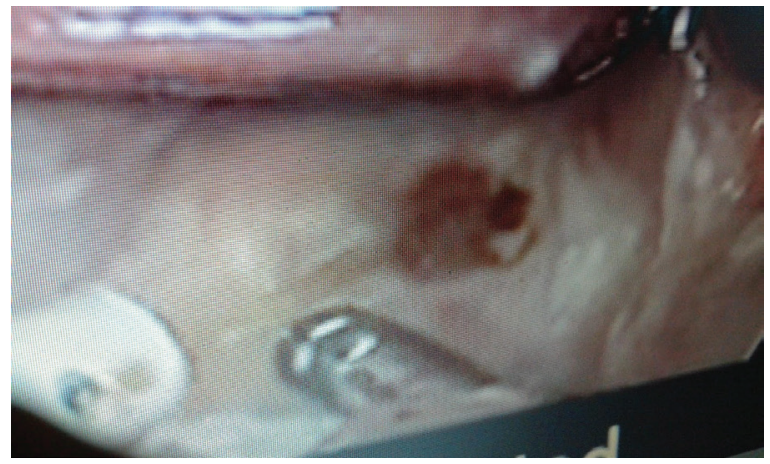

Figure (2): The site of the perforation.

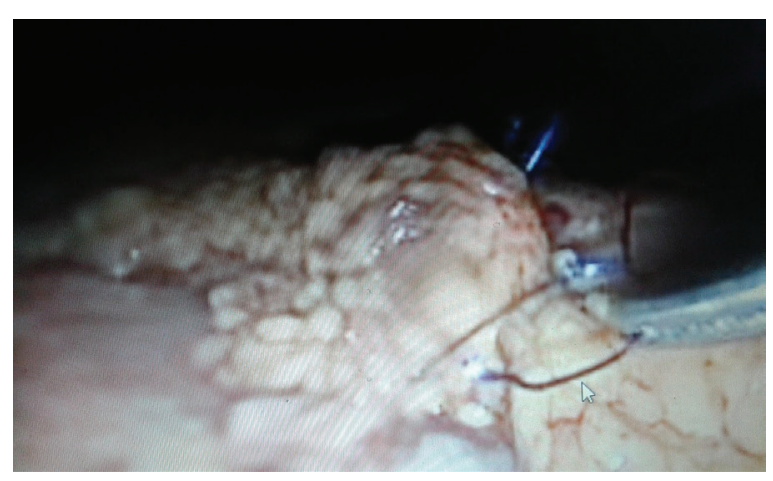

Figure (4): Omental patch over the sutured perforation.

adopted; we postponed the oral feeding for another 5 days.

The antibiotic was adjusted to Imipenem according to the culture and sensitivity results and the total parental nutrition was given. Radiological study was done on the $10^{\text {th }}$ day confirmed the healing of the perforation and the color of the drains was serous. The patient started oral feeding and discharged on the $12^{\text {th }}$ day.

The other case was 44 male patients, heavy smoker with history of peptic ulcer. On the $3^{\text {rd }}$ day postoperatively the patient complained of high fever, tachycardia and tachypnea. Chest $\mathrm{X}$-ray was done showed bilateral pneumonic patches and mild to moderate, right side pleural effusion. Definitive treatment was 
Table (1): Clinical characteristics of patients who underwent laparoscopic primary repair.

\begin{tabular}{|l|l|}
\hline \multicolumn{1}{|c|}{ Variables } & \multicolumn{1}{c|}{ Value } \\
\hline - Age & $37(19.0 \sim 55.0)$ \\
\hline - Sex & 16 \\
\hline Male & 4 \\
\hline Female & $29.5(17 \sim 42)$ \\
\hline -Body mass index $(\mathrm{kg} / \mathrm{m} 2)$ & 6 \\
\hline -History of treatment for duodenal ulcer \\
\hline Yes & 6 \\
\hline -Smoking consumption (per day) & 14 \\
\hline Over 20 cigarette: & 21 \\
\hline -Duration from symptom onset before surgery \\
\hline Below 24 hours & 4 \\
\hline Over 24 hours &
\end{tabular}

Table (2): Early surgical outcomes of laparoscopic primary repair.

\begin{tabular}{|l|l|}
\hline \multicolumn{1}{|c|}{ Variables } & \multicolumn{1}{c|}{ Value } \\
\hline Operation time (min) & $50( \pm 15)$ \\
\hline Overall postoperative complications & 2 \\
\hline Severe postoperative complications & $0.0(0.0)$ \\
\hline Day of commencement of soft diet & $5( \pm 3)$ \\
\hline Postoperative hospital stay (day) & $6( \pm 3)$ \\
\hline Conversion to open surgery & 2 \\
\hline
\end{tabular}

given for 7 days by the chest consultant after which the patient was discharged.

\section{Discussion:}

Despite recent controversies, laparoscopic surgery is likely to play an increasing role in the future. ${ }^{7}$

Nowadays laparoscopic procedures seems to be a useful method for reducing hospital stay, complications and return to normal activity if carried on in proper manner. Laparoscopy is attractive due to a lower morbidity rate associated with it than with conventional surgery. ${ }^{8}$ It reduces the access trauma, can confirm or refute the diagnosis, and can be used to perform the same repair procedure and lavage as open omental patch repair. 9

There are relative contraindication to the laparoscopic repair of perforated duodenal ulcer including delayed presentation (more than 24 hours from the onset of symptoms), comorbid disease and shocked patients. ${ }^{10}$

The study started after we had sufficient experience for the laparoscopic procedures, and it didn't not represent our initial experience in laparoscopic suture repair.

In our study, the analysis of surgical outcomes after laparoscopic primary repair showed positive surgical outcomes, similarly to the previous reports.

We had adopted the laparoscopic repair of the perforation using the intracoropreal stitches with the omental patch; we avoided the extracorporeal stitches as the edges of the perforation were friable and cannot withstand any traction.

Sutureless repair was once considered as safe as suture repair but it took extra cost such as the use of fibrin glue. Although the rationale of this sutureless technique was to simplify the procedure and shorten operative 
time, it did not gained wide acceptance owing to its high leakage rate as compared to suture repair $(16-6 \%) .{ }^{11}$

Turner et al., reported that suturing without an omental patch would result in a significantly higher mortality rate than with a patch. ${ }^{12}$

Our average operative time was 50 minuets which is average as compared with

(Chung H) and (Min Gyu Kim), ${ }^{7}$ and (Lau $\mathrm{H})$; (13) while it was shorter than (Lunevicius) and (Morkevicius M) who had claimed that laparoscopic repair with omental patch took up to 135 minutes. ${ }^{14}$

Conversion to open surgery was done in 2 cases $(10 \%)$ which were lower than Simone $\mathrm{G}$ et al., which was 30\% 15 and wing $\mathrm{T}$ et al which was $13 \% 16$ and average as Hoque et al. ${ }^{17}$

Suture leak represents one of the most frequent postoperative complications with a rate averaging from 5 to $16 \% .{ }^{18}$

In the present study, postoperative complications occurred in 2 patients, with 1 case of leakage $(5 \%)$. The leakage at the suture site was diagnosed by routine postoperative Gastrograffin study and clinical follow up; this patient was managed successfully by conservative treatment. The other patient had postoperative pneumonia that was managed the chest consultants.

We are confident that laparoscopic primary repair is a good method for the surgical treatment of duodenal ulcer perforation.

Laparoscopic repair of duodenal perforation seems to be a useful method for reducing hospital stay, complications and return to normal activity if carried on in proper manner. ${ }^{19}$

\section{Reference:}

1- Lau JY, Sung J, Hill C, Henderson C, Howden $\mathrm{CW}$, and Metz DC: Systematic review of the epidemiology of complicated peptic ulcer disease Incidence, recurrence, risk factors and mortality. Digestion 2011; 84: 102-113.

2- Bruner DI, Gustafson C: Respiratory distress and chest pain: A perforated peptic ulcer with an unusual presentation. Intern J Emerg Med 2011; 4: 34-38.

3- Furukawa A, Sakoda M, Yamasaki M, Kono
N, Tanaka T, Nitta N., et al: Gastrointestinal tract perforation: CT diagnosis of presence, site and cause. Abdom Imaging 2005; 30: 524-534.

4- Lau WY, Leow CK: History of perforated duodenal and gastric ulcers. World J Surg 1997; 21(8): 890-896.

5- Hainaux B, Agneessens E, Bertinotti R, Maertelaer V, Rubesova E, Capelluto E, et al: Accuracy of MDCT in predicting site of gastrointestinal tract perforation. AJR Am J Roentgenol 2006, 187: 1179-1183.

6- Ghekiere O, Lesnik A, Millet I, Hoa D, Guillon F, Taourel P: Direct visualization of perforation sites in patients with a nontraumatic free pneumoperitoneum: Added diagnostic value of thin transverse slices and coronal and sagittal reformations for multi-detector CT. Eur Radiol 2007; 17: 2302-2309.

7- Ma CH, Kim MG: Laparoscopic primary repair with omentopexy for duodenal ulcer perforation: A single institution experience of 21 cases. Journal of Gastric Cancer 2012, 12(4), 237-242.

8- Song KY, Kim TH, Kim SN, Park CH: Laparoscopic repair of perforated duodenal ulcers: The simple "one-stitch" suture with omental patch technique. Surg Endosc 2008; 22: 1632-1635.

9- Palanivelu C, Kalpesh J, Senthilnathan P: Laparscopic management of duodenal ulcer perforation: is it advantageous? Indian Journal of Gastroenterology 2007; 26: 64-66.

10- Matsuda M, Nishiyama M, Hanai T, Saeki S, Watanabe T: Laparoscopic omental patch repair for perforated peptic ulcer. Annals of Surgery 1995; 221(3): 236-240.

11- Druart ML, Van Hee R, Etienne J, Cadière GB, Gigot JF, Legrand M, et al: Laparoscopic repair of perforated duodenal ulcer: A prospective multicenter clinical trial. Surg Endosc 1997; 1017-1020.

12- Turner WW, Thompson WM, Thal ER: Perforated gastric ulcers. A plea for management by simple closures. Arch Surg 1998; 123: 960-964.

13- Lau H: Laparoscopic repair of perforated peptic ulcer. Review article. Surg Endosc 2004; (18) 1013-1021.

14- Lunevicius R, Morkevicius M: Systematic review comparing laparoscopic and open repair of perforated peptic ulcer. Br J Surg 2005; (92) 1195-1200.

15- Simone G, Ismail C, Christian G, Niccolò F, Vincenzo L, Giuseppe Z, et al: Laparoscopic 
repair of perforated peptic ulcer: Singlecenter results. Surgical Endoscopy 2014; (28) 2302-2308.

16- Wing T, Heng T, Bonita K, Chun H, Anthony C, Kai $H$, et al: Laparoscopic repair for perforated peptic ulcer: A randomized controlled trial. Ann Surg 2002; 235(3): 313-319.

17- Hoque U, Hossain ZN, Bhuain NH, HasanR, Mahmud U: Laparscopic repair of peptic ulcer perforation - our initial experience.
Journal of Pediatric Surgeons of Bangladesh 2010; 1(2): 153-157.

18- Lee FY, Leung KL, Lai PB, Lau JW: Selection of patients for laparoscopic repair of perforated peptic ulcer. Br J Surg 2001; 88(1): 133-136.

19- Luncă, Romedea N S, Moroşanu C: Laparoscopic Repair of perforated peptic ulcer. Jurnalul de Chirurgie 2007; (3) 1584-9341. 